\title{
POINT-SPECTRUM OF SEMIBOUNDED OPERATOR EXTENSIONS
}

\author{
PALLE E. T. JØRGENSEN ${ }^{1}$
}

\begin{abstract}
Let $\tilde{H}$ denote the Friedrichs extension of a given semibounded operator $H$ in a Hilbert space. Assume $\lambda l<H$, and $\lambda \in \sigma(\tilde{H})$. If for a finite-dimensional projection $P$ in the Hilbert space we have $I-P<$ Const. $(H-\lambda I)$, then it follows that $\lambda$ is an eigenvalue of $\tilde{H}$, and the corresponding eigenspace is contained in the range of $P$. Using this, together with the known order structure on the family of selfadjoint extensions, with given lower bound 0 , of minus the Laplace-Beltrami operator, we establish the identity $U_{g}(1)=1$ for all $g \in G$ for the following problem.

$U$ is a unitary representation of a Lie group $G$, and acts on the Hilbert space $L^{2}(\Omega)$ for some Nikodym-domain $\Omega \subset G$. Moreover $U$ is obtained as a certain normalized integral for the left- $G$-invariant vector fields on $\Omega$, that is, for each such vector field $X$, the skew-adjoint operator $d U(X)$ is an extension of $X$ when regarded as a skew-symmetric operator in $L^{2}(\Omega)$ with domain $C_{0}^{\infty}(\Omega)$.
\end{abstract}

1. Introduction. Recently, two results, related to the spectral theory of the Friedrichs extension, have appeared. In [7] it was shown that the SegalFuglede integration problem for constant coefficient partial differential operators [1] has an affirmative answer in a more general setting of Lie groups and symmetric spaces. The answer relates the geometry of a given finite-volume domain $\Omega$ to the integration problem for Lie algebras of invariant vector fields on $\Omega$. However, the spectral theory of the corresponding Laplace-Beltrami operators (and their extensions) is still not well understood. (Independently, in [6] it is established that the Friedrichs extension of a semibounded operator $\boldsymbol{H}$ has derivation properties with respect to a given operator algebra if it is known that the initial nonselfadjoint operator $\boldsymbol{H}$ has these properties.)

The following theorem has implications for both of the above-mentioned problems, and it seems to be of independent interest (in view of the scarcity of general results on the point spectrum of the Friedrichs extension). The idea of the proof is based on that of Fuglede's main lemma [1], which is in fact about families of selfadjoint nonsemibounded operators. The results however have little in common.

\section{Ground states.}

THEOREM 1. Let $H$ be a semibounded symmetric operator in a Hilbert space $\mathfrak{E}$, and let $\tilde{H}$ denote the Friedrichs extension of $H$. Let $P$ be a finite-dimensional projection in

Received by the editors March 27, 1980.

1980 Mathematics Subject Classification. Primary 47A70, 47D40; Secondary 81C10.

Key words and phrases. Estimates for operators, extensions, eigenvalues.

${ }^{1}$ Research supported in part by NSF Grant MCS-78-02942. 
$\mathcal{L}, C$ a positive constant, and let $\lambda$ be in the spectrum of $\tilde{H}$. Suppose

$$
I-P<C(H-\lambda) \text {. }
$$

Then $\lambda$ is an eigenvalue of $\tilde{H}$, and the corresponding eigenspace is contained in the range of $P$.

Proof. The inequality (1) refers to the usual order on symmetric operators. It translates into the estimate $\langle u-P u, u\rangle=\|u-P u\|^{2}\langle C\langle H u-\lambda u, u\rangle$ for all vectors $u$ in the dense domain $\mathscr{D}(H)$ of $H$.

Let $\tilde{H}=\int \xi d E_{\xi}$ denote the canonical spectral representation of $\tilde{H}$. Set $\delta_{k}=\{\xi$ $\in \mathbf{R}:|\xi-\lambda|<1 / k\}$. The assumption $\lambda \in \sigma(\tilde{H})$ implies $E\left(\delta_{k}\right) \neq 0$ for all $k=$ $1,2, \ldots$. Pick vectors $u_{k} \in E\left(\delta_{k}\right) \mathcal{L},\left\|u_{k}\right\|=1$. By the definition of $\tilde{H}$ there is a real number $r$ such that $H+r \geqslant I$. For each $k$ there is a sequence $u_{j k} \in \mathscr{D}(H)$ such that

$$
\lim _{j}\left\langle(H+r) u_{j k}, u_{j k}\right\rangle=\left\langle(\tilde{H}+r) u_{k}, u_{k}\right\rangle \text { : }
$$

By (1) we get $\left.\left\|u_{j k}-P u_{j k}\right\|^{2}<C<(H-\lambda) u_{j k}, u_{j k}\right\rangle$. Passing to the limit $j \rightarrow \infty$ we get

$$
\left\|u_{k}-P u_{k}\right\|^{2} \leqslant C\left\langle(\tilde{H}-\lambda) u_{k}, u_{k}\right\rangle=C \int(\xi-\lambda)\left\langle d E_{\xi} u_{k}, u_{k}\right\rangle\langle C 1 / k .
$$

In fact formula (1) is valid with $H$ replaced by $\tilde{H}$.

By weak compactness of the unit-sphere in $\mathcal{L}$ there is a subsequence $u_{k}^{\prime}$ of $u_{k}$ which is weakly convergent with limit $u$ say, such that $\|u\|=1$. By (3) we also have $\left\|u_{k}^{\prime}-P u_{k}^{\prime}\right\| \rightarrow 0$; and since $P$ is finite-dimensional, $\left\|P u_{k}^{\prime}-P u\right\| \rightarrow 0$. Hence

$$
\left\|u_{k}^{\prime}-P u\right\| \rightarrow 0,
$$

and we conclude that $u$ belongs to the finite-dimensional range of $P$; that is to say $P u=u$. Substitution into (4) gives $\left\|u_{k}^{\prime}-u\right\| \rightarrow 0$. We have

$$
\left\|(\tilde{H}-\lambda) u_{k}^{\prime}\right\|^{2}=\int|\xi-\lambda|^{2}\left\langle d E_{\xi} u_{k}^{\prime}, u_{k}^{\prime}\right\rangle \rightarrow 0
$$

since the integration is over intervals centered at $\lambda$ of radius $\rightarrow 0$. We conclude $u \in \mathscr{D}(\tilde{H})$ and $\tilde{H} u=\lambda u$.

If, conversely, the identity $\tilde{H} u=\lambda u$ is known to hold, then (1), with $\tilde{H}$ in place of $H$, implies $P u=u$.

The assumption (1) in the theorem implies in particular the operator estimate $\lambda I \leqslant \tilde{H}$. Hence the conclusion states "existence of ground states" [4] and, if $P$ is a priori known to be one-dimensional, also uniqueness. In the special case where $H$ is known to be selfadjoint and $\lambda=\inf \sigma(H)$ the theorem yields that $\lambda$ is in fact an eigenvalue.

Now let $H \geqslant 0$ be an arbitrary selfadjoint extension of a given symmetric operator $L \geqslant 0$. Let $\tilde{H}$ be the Friedrichs extension of $L$, and consider the conditions (a) $0 \in \sigma_{p}(\tilde{H})$, and (b) $0 \in \sigma_{p}(H)$.

An application of Krein's theory for semibounded operators [10] yields the implication (a) $\Rightarrow(b)$. Note that both of the conditions (a) and (1) can be stated in 
terms of estimates only on the vectors in the domain of $L$. In applications below, (b) will be satisfied in cases where (a) is not. As a compensation the estimate (1) will be known to hold on the domain for a certain selfadjoint extension $H$, rather than just on $\mathscr{D}(L)$.

3. The Laplace-Beltrami operator. Let $M$ be a Riemannian $\left(C^{\infty}-\right)$ manifold, and let $\Omega \subset M$ be an open connected subset with finite Riemannian measure. If $\bar{\Omega}$ is noncompact, the point-spectrum of the Laplace-Beltrami operator $\Delta$ is not well understood [9]. We say that $\Omega$ is a Nikodym-domain [2] if there is a (finite) constant $C$ such that

$$
\int_{\Omega}\left|u(x)-\left(\int_{\Omega} u\right) m(\Omega)^{-1}\right|^{2} d x<C \int_{\Omega}|\operatorname{grad} u|^{2} d x
$$

for all $u \in \mathcal{H}^{1}(\Omega)$. Here the integration is with respect to the Riemannian density $d x$, the constant $C$ is the Poincare constant, grad is the gradient defined from the given Riemannian metric [5], and finally the notation $\mathcal{K}^{1}(\Omega)$ designates the first $L^{2}$ (inhomogeneous) Sobolev space on $\Omega$.

Corollary 2. Let $\Omega \subset M$ be a Nikodym-domain as defined above, and let $H$ be a selfadjoint extension of $-\Delta$ on $C_{0}^{\infty}(\Omega)$, with $0=$ inf $\sigma(H)$, regarded as an operator in $\mathcal{L}=L^{2}(\Omega)$. Assume the inclusion $\mathscr{D}\left(H^{1 / 2}\right) \subset \mathcal{H}^{1}(\Omega)$ where $\mathscr{D}\left(H^{1 / 2}\right)$ denotes the domain of the square root operator. Then $\lambda=0$ is an eigenvalue of $H$ with one-dimensional eigenspace $\mathrm{C} 1$.

Proof. If $P$ denotes the $L^{2}(\Omega)$-orthogonal projection onto the constant function 1, then condition (5) implies the estimate (1) for $H$ with $\lambda=0 \in \sigma(H)$. To see this we note that $(H u, u)=\left\|H^{1 / 2} u\right\|^{2}=\|\operatorname{grad} u\|_{0}^{2}$ for $u \in C_{0}^{\infty}(\Omega)$. If $H_{N}$ denotes the s.a. extension of $-\Delta$ with homogeneous Neumann conditions, then an application of Green's identity leads to the inequality

$$
\int_{\Omega}|\operatorname{grad} u|^{2} d x=\left\|H_{N}^{1 / 2} u\right\|^{2}<\left\|H^{1 / 2} u\right\|^{2}
$$

for $u \in \mathscr{D}\left(H^{1 / 2}\right) \subset \mathscr{D}\left(H_{N}^{1 / 2}\right)$. When this is combined with (5), the desired inequality (1) follows.

4. Invariant vector fields. The problem of invariant vector fields which was mentioned in the introduction was posed in the Euclidean case first by I. E. Segal [1], [8], and formulated in the general case by the author in [7], (motivated in turn by [1]). There a fixed Lie group $G$ with Haar measure $m$ is considered, along with an open subset $\Omega \subset G, 0<m(\Omega)<\infty$. The existence of a unitary representation $U$ of $G$ on $L^{2}(\Omega)$ is assumed such that, for all elements $X$ in the Lie-algebra $\mathcal{G}$ of left-invariant vector fields, the selfadjoint generator $d U(X)$ extends the vector field $-i X$ regarded as a symmetric operator in $L^{2}(\Omega)=\mathcal{L}$ [5]. In the case where the action of $U(G)$ is multiplicative on $\mathcal{E}$, it is shown in [7] that the integrable domains $\Omega$ (i.e. those which admit a unitary representation $U$ with the above infinitesimal extenson property) are precisely those which, after correction on a null set, form a 
fundamental domain for a discrete subgroup of $G$. In the general case of nonmultiplicative action, the subgroup $A=\left\{a \in G: U_{a} \mathfrak{N} U_{a}^{*}=\mathfrak{N}\right\}$ enters in an imprimitivity system for the maximal abelian $L^{\infty}(\Omega)$-multiplication algebra $\Re$ on $L^{2}(\Omega)$, [7], [8]. If $U_{g}(1)=1$ for all $g \in G$, it follows that $A=\left\{a \in G: U_{a}\right.$ acts multiplicatively\}, and hence that Mackey's theory applies [8].

TheOREM 3. Let $\Omega$ be an integrable Nikodym-domain in a connected Lie group $G$, and let $U$ be a unitary representation of $G$ on $L^{2}(\Omega)$ satisfying the infinitesimal extension property with respect to the invariant vector fields defined on $C_{0}^{\infty}(\Omega)$. For a basis $X_{1}, \ldots, X_{d}$ in $\mathcal{G}$ assume that $0 \in \sigma\left(d U\left(\sum X_{i}^{2}\right)^{-}\right)$. Then $U_{g}(1)=1$ for all $g \in G$. Moreover only the constant functions are left fixed by all the operators $U_{\boldsymbol{g}}$.

Proof. It is known that the operator $H=d U\left(\Sigma X_{i}^{2}\right)^{-}>0$ is selfadjoint on $\mathcal{L}=L^{2}(\Omega)[12]$ and the assumption in the theorem implies that $H$ is an extension of $-\Delta$ where $\Delta$ is the Laplace-Beltrami operator, corresponding to the given basis $X_{i}$ and the invariant Riemannian metric on $G$. Let $C^{1}(U) \subset \mathcal{L}$ denote the $C^{1}$-vectors [3] for $U$. Then $\mathscr{D}\left(H^{1 / 2}\right)=C^{1}(U) \subset \mathcal{H}^{1}(\Omega)$. Indeed the equality on the left is [3, Proposition 1.3], and the inclusion $\subset \mathcal{H}^{1}(\Omega)$ may be derived as follows. For $u \in C^{1}(U)$ the mapping $g \rightarrow U_{g}(u)$ is of class $C^{1}$ on $G$ as a $\mathcal{L}$-valued vector function. For each $X \in \mathcal{G}$ let $\tilde{X}$ denote the distribution action of $X$. The assumption in the theorem implies that $d U(X)(u)=\tilde{X}(u) \in \mathcal{L}=L^{2}(\Omega)$ for all $X \in \mathcal{G}$. This means in turn that $u \in \mathcal{H}^{1}(\Omega)$, by the very definition of this first Sobolev space.

The assumptions of Corollary 2 are satisfied, and the conclusion $1 \in \mathscr{D}(H)$ holds. Hence $\Sigma\left\|d U\left(X_{i}\right) 1\right\|^{2}=(H 1,1)=(-\tilde{\Delta} 1,1)=0$. It follows by differentiation that the function $g \rightarrow U_{g}(1)$ is locally constant, and hence, by connectedness of $G$, constant. The conclusion $U_{g}(1)=1$ follows by evaluation at $g=e$.

If conversely $U_{g}\left(u_{0}\right)=u_{0}$ for all $g \in G$, then $u_{0} \in C^{\infty}(U)$ and $H\left(u_{0}\right)=$ $\Sigma d U\left(X_{i}\right)^{2}\left(u_{0}\right)=0$. But the 0 -eigenspace for $H$ is, by Corollary 2 , spanned by the constant function 1 , so that $u_{0}$ must be constant.

REMARK. Note that the theorem applies in particular, and the conclusion holds, if $\Omega$ is relatively compact in $G$. But then the result would also follow by RellichSobolev compactness [13]. In recent applications, see for example [11], we know that $\bar{\Omega}$ is not compact, but $m(\Omega)<\infty$ is satisfied.

Added in proof. Reference [8] has been announced by P. Jørgensen in Proc. Nat. Acad. Sci. U.S.A. 77 (1980), 5050-5051. Theorem 1 has been generalized by the author to operators in Banach space which generate one-parameter groups of isometries. Preprints available.

\section{REFERENCES}

1. B. Fuglede, Commuting self-adjoint partial differential operators and a group theoretic problem, J. Funct. Anal. 16 (1974), 101-121.

2. J. Deny and J. L. Lions, Les espaces du type de Beppo Levi, Ann. Inst. Fourier (Grenoble) 5 (1953-54), 305-370.

3. R. Goodman, One-parameter groups generated by operators in an enveloping algebra, J. Funct. Anal. 6 (1970), 218-236. 
4. L. Gross, Existence and uniqueness of physical ground states, J. Funct. Anal. 10 (1971), 52-109.

5. S. Helgason, Differential geometry and symmetric spaces, Academic Press, New York, 1962.

6. P. E. T. Jorgensen, Extensions of symmetric operators and unbounded derivations, J. Math. Anal. Appl. 73 (1980), 115-133.

7. __ Partial differential operators and discrete subgroups of a Lie group, Math. Ann. 247 (1980), 101-110.

8. Spectral theory of finite volume domains in $\mathbf{R}^{n}$, Adv. in Math. (to appear).

9. H. Kalf, On the characterization of the Friedrichs extension at ordinary or elliptic differential operators with a strongly singular potential, J. Funct. Anal. 10 (1972), 230-250.

10. M. G. Krein, The theory of self-adjoint extensions of semi-bounded hermitian operators and its applications, Mat. Sb. 20 (62) (1947), 431-495.

11. P. D. Lax and R. S. Phillps, Scattering theory for automorphic functions, Ann. of Math. Studies, vol. 87, Princeton Univ. Press, Princeton, N. J., 1976.

12. E. Nelson and W. F. Stinespring, Representation of elliptic operators in an enveloping algebra, Amer. J. Math. 81 (1959), 547-560.

13. F. Rellich, Ein Satz über mittlere Konvergenz, Nachr. Acad. Wiss. Göttingen Math.-Phys. K1.II (1930), 30-35.

Mathematics Institute, Aarhus University, 8000 Aarhus C, Denmark 